\title{
RD50: Radiation-Hard Silicon for HL-LHC Trackers
}

\section{Monica Scaringella ${ }^{1 *}$}

University of Florence

Via S. Marta 3, Firenze, Italy

E-mail: scaringellaefi.infn.it

To harvest the maximum physics potential of the LHC, it is foreseen to significantly increase the luminosity by upgrading towards the HL-LHC (High Luminosity LHC). This will mean unprecedented radiation levels, exceeding the LHC fluences tenfold. Due to radiation damage to the silicon sensors presently used, the physics experiments will require new tracking detectors. Within the CERN RD50 Collaboration, a massive R\&D programme is underway across experimental boundaries to develop HL-LHC silicon sensors. One research topic is the connection between the macroscopic sensor properties such as radiation-induced increase of leakage current, doping concentration and trapping, and the microscopic properties. We also study sensors made from p-type silicon bulk, which have a superior radiation hardness as they collect electrons instead of holes, exploiting the lower trapping probability of the electrons and allows for operation in partial depletion. A selection of the latest results achieved within the collaboration on silicon detectors irradiated at levels corresponding to HL-LHC fluences is reported in this paper.

10th International Conference on Large Scale Applications and Radiation Hardness of Semiconductor Detectors Firenze, Italy

July 6-8, 2011

* on behalf of the RD50 collaboration 


\section{Introduction}

The CERN RD50 Collaboration has, as a main focus, the development of super-radiation hard detectors for applications in high energy physics experiments characterized by very high luminosities, with particular regard to future LHC upgrades (High Luminosity LHC: HL-LHC) [1]. For the CMS [2] and ATLAS [3] experiments, the tenfold luminosity increase foreseen for the HL-LHC [4] will translate into radiation levels of about $2 \times 10^{16} \mathrm{n}_{\mathrm{eq}} / \mathrm{cm}^{2}$ for the innermost pixel layers and $1 \times 10^{15} \mathrm{n}_{\mathrm{eq}} / \mathrm{cm}^{2}$ for the innermost strip layers. In the present paper the particle fluence is normalised to the damage of neutrons with $1 \mathrm{MeV}$ energy according to the NonIonising Energy Loss (NIEL) model [5]. The technological challenges posed by the HL-LHC radiation levels are related to radiation damage effects deteriorating the detectors performances. The first one is the increase of the reverse current in the sensor, which is proportional to the particle fluence normalised to the damage of $1 \mathrm{MeV}$ neutrons. This effect is mainly due to the production of generation-recombination centres by irradiation. The second effect is the change of the effective doping concentration of the sensor, $\mathrm{N}_{\mathrm{eff}}$, due to the progressive removal of the intentional shallow dopants and the generation of deep energy levels, strongly influencing the full depletion voltage and the electric field profile within the device. Due to this effect, an nbulk sensor can be transformed to a quasi-intrinsic p-bulk by radiation [6]. Nevertheless, the phenomenon is strongly dependent on the particle irradiation kind and on the type of material used. The increasing doping concentration manifests itself as an increasing full depletion voltage of the sensor, which may at a certain fluence exceed either the voltage tolerance of the detector or the maximal voltage rating of the High Voltage (HV) power supply. The third effect is the introduction of defects that can trap/recombine drifting charges in the silicon bulk, thus effectively reducing the charge collection efficiency (CCE) of the sensor. The radiationinduced trapping of carriers results in a reduction of the signal measured with the sensor, and hence reduces the signal-to-noise ratio of the detector. This effect is worsened by the growing noise originating from extra shot noise due to the additional leakage current generated from radiation damage.

\section{Scientific strategies}

Main approaches of radiation hardening developed by the collaboration to cure these unwanted effects are based on both material and device engineering. Material engineering mainly relies on opportunely tailoring the material properties of the semiconductor, e.g. through intentional doping, in view to control main electric transport parameters, to increase the charge collection efficiency of the irradiated devices and their operative lifetime. Main investigations are devoted to n- and p-type crystalline silicon growth by Float Zone (FZ) technique, Diffusion Oxygenated FZ (DOFZ), characterized by an enhanced concentration of oxygen, as well as Czochralski (Cz), magnetic $\mathrm{Cz}(\mathrm{MCz})$ and epitaxial silicon [7]. The second main research line, "Device Engineering", is aimed at developing more radiation-tolerant detector geometries than standard ones. To progress along these two research lines, design, manufacturing and a systematic characterization of both materials and devices before and after irradiation with 
radiation fields and fluencies close to the operative ones have been carried out by the collaboration through many years. A selection of the most recent results achieved within the collaboration is presented and discussed in this paper.

\section{Charge collection efficiency}

This section will focus on some of the most significant results on radiation damage achieved within the collaboration with n-on-p silicon sensors in terms of CCE. One of the main advantages of this type of sensors, with respect to the standard p-on-n devices, is that they are based on electrons read-out, so they can benefit from the fact that electrons have higher mobility in silicon with respect to holes. The higher performance of devices with n-side read-out was already proven by n-on- $n$ technology [8], in addition n-on-p devices have the advantage that can be produced by single-side processing and thus at lower cost.

Several studies on charge collection for p-type segmented detectors performed in the last few years have demonstrated that a significant amount of charge can be measured after irradiation at very high hadron fluences (of the order of $10^{16} \mathrm{n}_{\mathrm{eq}} / \mathrm{cm}^{2}$ ) [9-15], indicating that planar p-type detectors technology can be considered as an option for the inner layers of HLLHC detectors. It has also been observed that the collected charge for these devices at those fluences is higher than expected from the extrapolation of radiation damage parameters, like full depletion voltage and trapping times, measured at fluences up to $10^{15} \mathrm{n}_{\mathrm{eq}} / \mathrm{cm}^{2}[13,15]$. As an example, figure 1 [9] shows the results of collected charge measurements performed on n-on-p $300 \mu \mathrm{m}$ thick FZ Si sensor after irradiation with $24 \mathrm{GeV}$ and $26 \mathrm{MeV}$ protons and with reactor neutrons, at $900 \mathrm{~V}$ reverse bias, as a function of $\mathrm{n}_{\mathrm{eq}}$ fluence. The measurements were performed using a ${ }^{90} \mathrm{Sr}$ beta source and a SCT128A [16] analogue readout ASIC clocked at $40 \mathrm{MHz}$. After the maximum fluence a charge above 5000 electrons can still be collected.

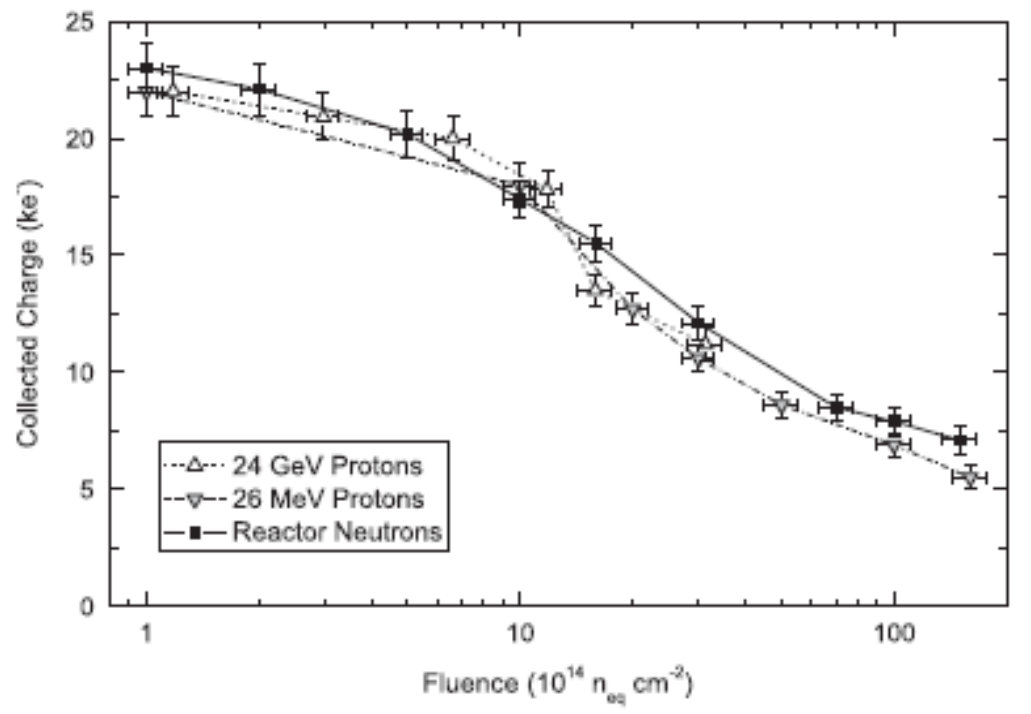

Fig.1 Charge collection as a function of $n_{\text {eq }}$ fluence measured on irradiated $n$ on p $300 \mu \mathrm{m}$ FZ detectors at $900 \mathrm{~V}$ reverse bias [9]. 
Moreover some of the charge collection measurements on highly irradiated sensors at very high bias voltages show a collected charge as high or even higher with respect to unirradiated devices [13-15]. In figure 2 charge collection measurements as a function of reverse bias on non-p detectors irradiated with $26 \mathrm{MeV}$ protons up to $5 \times 10^{15} \mathrm{n}_{\mathrm{eq}} / \mathrm{cm}^{2}$ are reported. The experimental set-up for the charge collection is the same used for the measurements reported in figure 1. It can be observed that for $140 \mu \mathrm{m}$ thick samples the collected charge can be significantly higher than the ionized charge by a mip in $140 \mu \mathrm{m}$ of silicon $(\sim 11.2 \mathrm{ke})$. This result can only be explained in terms of multiplication effects on the generated charge in high field regions inside the silicon bulk.

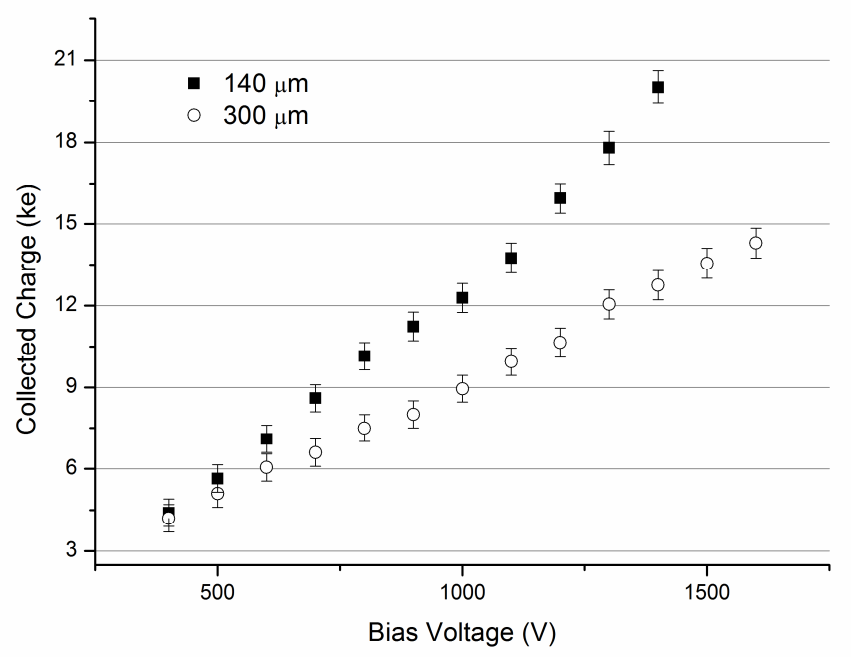

Fig.2 Collected charge measured on n-on-p FZ detectors irradiated with $26 \mathrm{MeV}$ protons up to $5 \times 10^{15} \mathrm{n}_{\mathrm{eq}} / \mathrm{cm}^{2}$ as a function of bias voltage, data from [14].

\section{Electric Field distribution}

The enhanced collected charge measured with $\mathrm{n}$ on $\mathrm{p}$ silicon sensor has motivated the study on the electric field distribution inside the bulk of this kind of devices in order to understand the possible mechanisms underlying the observed phenomena and to investigate the possibility of operating the devices in charge multiplication regime. This study has been conducted both with simulations and with measurements, in this section some of the results of these investigations will be reported.

\subsection{Modeling of charge multiplication effect}

One of the mechanisms invoked to explain the observation of CCE greater than 1, for highly irradiated detectors at high bias voltage, is that in some region of the bulk the electric field reaches levels high enough to produce the onset of avalanche multiplication. On the other hand, once this effect is established, one would expect a sharp increase of the CCE versus voltage characteristics, which is not observed as it can be also noted in figure 2 . In order to explain this behavior a model has been proposed that takes into account of avalanche 
multiplication and trapping by deep levels. In this section a brief report of the model will be given, more details can be found in reference [17]. For highly irradiated detectors the electrical characteristic are completely dominated by the radiation induced deep levels that can act as traps, dopants and recombination/generation centers. The influence of deep levels on the onset of avalanche multiplication is schematically shown in figure 3 where the electric field and the current density as a function of the depth inside the sample are reported in three different cases: low field state ( $a$ and $b$ ), high field non-steady state ( $c$ and $d$ ) and high field steady state ( $\mathrm{d}$ and f). A linear behavior of the electric field inside the space charge region (SCR) is assumed. We assume to start in a low field state where no multiplication occurs, in this case the current density is given by the electrons and holes thermally generated in the bulk. At some point the bias voltage is increased to a value at which in a narrow region towards the junction (indicated by the grey area in figure 5c) the electric field level is high enough to produce avalanche multiplication. This state is non-steady since the avalanche electron multiplication is associated to an avalanche hole injection inside the bulk: in this case the hole current density is given by the bulk generated holes and the avalanche hole injection contribution. A fraction of these holes will be trapped by deep levels inside the bulk giving rise to a positive space charge that produces an increase of the depletion depth and thus a decrease of the electric field level at the junction (high field steady-state). This means that the presence of deep levels somehow gives rise to a negative feedback that stabilizes the avalanche multiplication, prevents the detector breakdown and smoothes out the CCE-V and I-V characteristics.
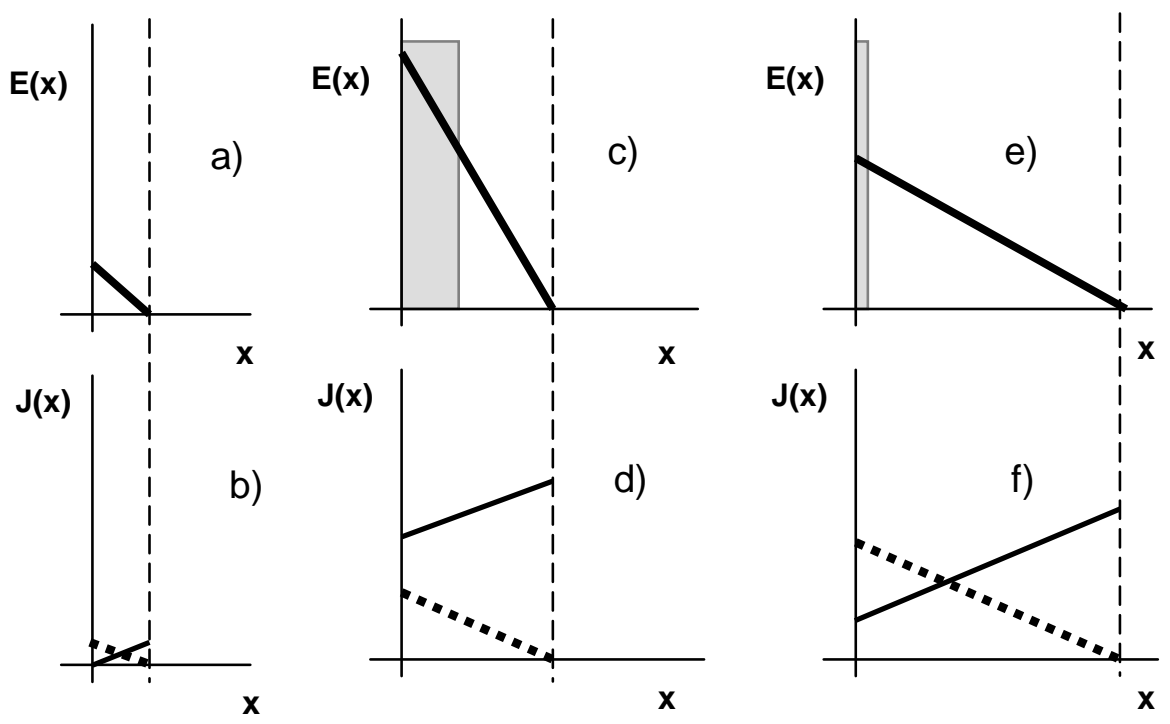

Fig.3 Electric field and current density profiles in low field steady state ( $a$ and $b$ ) high field nonsteady state (c and d) and high field steady state (e and f). Dotted and solid lines in the current density plots indicate the electron and hole current density components respectively [17].

\subsection{Edge Transient Current Technique}

The Edge Transient Current Technique (Edge TCT) is an experimental method that has been introduced in order to estimate the electric field distribution along the thickness of the sample. The difference with respect to the conventional TCT method is that the sample is 
illuminated from the edge by a narrow infrared laser (1060 nm wavelength) that can be focused underneath the reading strip and scanned along the detector thickness so that the charge is generated only in a small region at a certain depth. At each depth the current transient generated by the illumination is then sampled by a high bandwidth oscilloscope. From the analysis of the current transients the drift velocity profile, which in turn gives an indication of the electric field profile, can be extracted according to the methods described in [18]. The profile of the collected charge can be simply extracted by integrating the current transient over time.

In figure 4 [19] the profile of the collected charge extracted from edge-TCT measurements performed at $700 \mathrm{~V}$ bias voltage for an n-on-p sensor irradiated with reactor neutrons at $10^{15}$ $\mathrm{n}_{\mathrm{eq}} / \mathrm{cm}^{2}$ and annealed at $60{ }^{\circ} \mathrm{C}$ for various annealing times is shown.

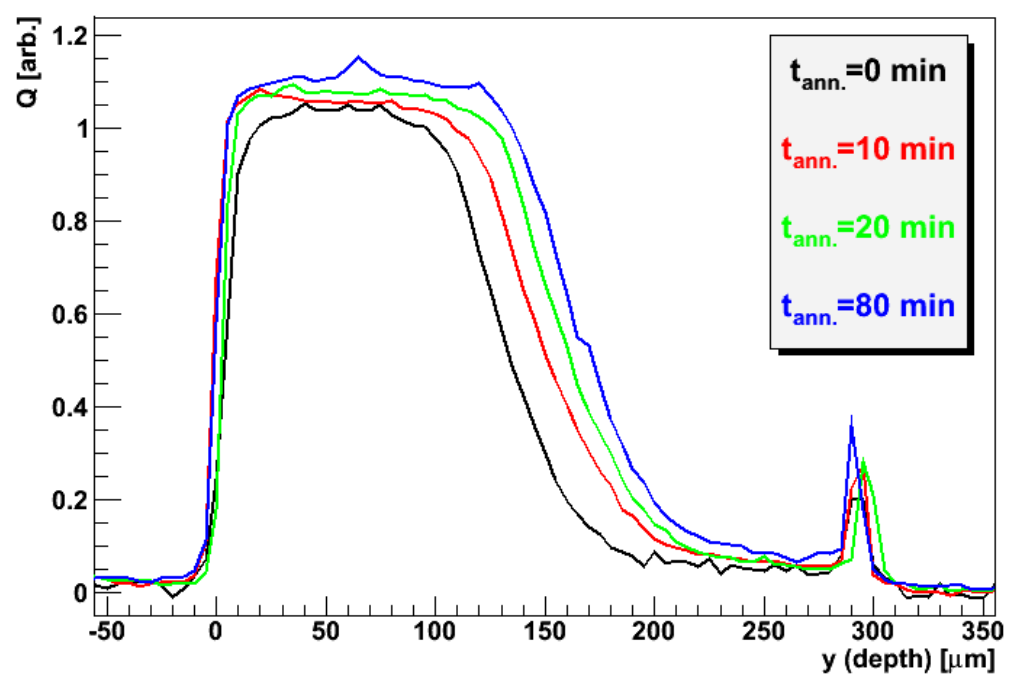

Fig.4 Profile of the collected charge extracted from Edge-TCT measurements performed at $700 \mathrm{~V}$ bias voltage for an $\mathrm{n}$ on $\mathrm{p}$ sensor irradiated with reactor neutrons up to $10^{15} / \mathrm{cm}^{2}$ and annealed at 60 ${ }^{\circ} \mathrm{C}$ for various annealing times [19].

It can be observed how the charge is only collected from a portion of the thickness (active area) while the charge collected by the remaining part (undepleted area) is negligible. Also the beneficial annealing effect is visible and a peak at the back of the device indicating the presence of the double junction effect [20]. The depletion depth corresponding to the $\mathrm{N}_{\text {eff }}$ calculated from the Hamburg model [21] for this sample after 80 minutes annealing at $60{ }^{\circ} \mathrm{C}$ is $200 \mu \mathrm{m}$ at 700 $\mathrm{V}$, value that correlates very well with the behavior of the collected charge profile. This indicates that at this fluence the device behave in accordance to modeling derived at lower fluences. In figure 5 [19] the drift velocity and collected charge profiles for a similar sample irradiated at $10^{16} \mathrm{~cm}^{-2}$ are shown. In this case it can be noticed that the distinction between active and non-active region loses significance and the electric field establishes over the whole thickness. This means that the "active region" that one would extract from depletion voltage measurements underestimates the effective collected charge and can explain, together with the charge multiplication effect, the enhanced CCE for this kind of devices. 

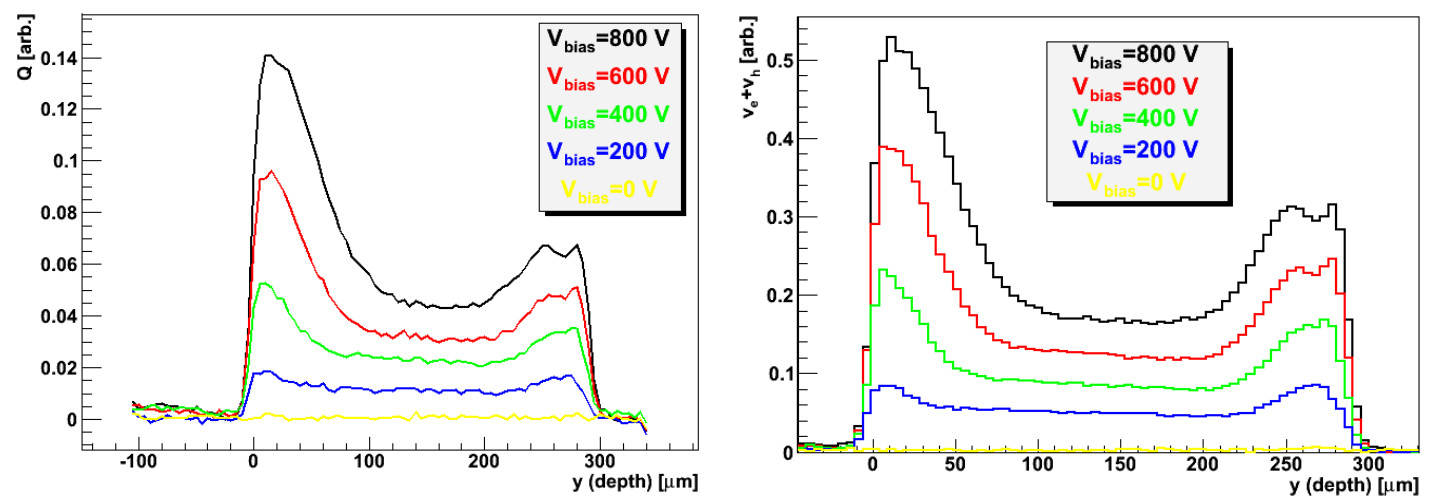

Fig.4 Collected charge and drift velocity profiles extracted from Edge-TCT measurements performed at various bias voltage for an $\mathrm{n}$ on $\mathrm{p}$ sensor irradiated with reactor neutrons up to $10^{16}$ $\mathrm{cm}^{-2}[19]$.

\section{Conclusions}

The RD50 collaboration is working on the development of super radiation-hard detectors for high luminosity colliders such as HL-LHC, the foreseen upgrade of LHC. Charge collection measurements performed on FZ p-type silicon detectors irradiated at very high hadron fluence show very good performance of these devices when operated at very high voltage; the signal value is higher than expected by calculations based on radiation damage parameters extracted from lower fluence measurements. In some cases the value of the measured charge exceeds the one measured on unirradiated detectors, this effect can only be explained in terms of charge multiplication occurring in high field regions of the devices. A model has been developed to explain the self-stabilizing effect of electron avalanche multiplication occurring towards the $\mathrm{n}^{+}$ junction, due to the high concentration of deep levels. The evaluation of the electric field distribution along the thickness of the sample performed through Edge TCT measurements has evidenced that the modeling of depleted thickness using parameters derived at low fluences underestimates the collected charge because the electric field establishes over the whole thickness of the device. This effect, together with the charge multiplication effect, explains the enhanced charge collection of highly irradiated p-type silicon detectors.

\section{References}

[1] RD50 Collaboration, RD50 Status Report 2008, CERN-LHCC-2010-012 and LHCC-SR-003.

[2] CMS Collaboration, JINST 3 (2008) S08004.

[3] ATLAS Collaboration, JINST 3 (2008) P07007.

[4] F. Ruggiero, Eur Phys J C 34 (suppl. 1) (2004) s433.

[5] M. Huhtinen, Nucl. Instr. and Meth. A 491 (2002) 194.

[6] U. Biggeri, E. Borchi, M. Bruzzi, S. Lazanu, Nucl. Instr. and Meth. A 360 (1995) 131. 
[7] M. Bruzzi, et al., Nucl. Instr. and Meth. A 541 (2005) 189.

[8] Y. Unno, et al. IEEE Trans. Nucl. Sci. 46 (1999) 1957.

[9] A. Affolder, et al. Nucl. Instr. and Meth. A 612 (2010) 470.

[10] G. Casse, A. Affolder and P. P. Allport, IEEE Trans. Nucl. Sci. 55 (2008) 1695.

[11] G. Casse, A. Affolder, P. P. Allport and M. Wormald, IEEE Trans. Nucl. Sci. 56 (2009) 3752.

[12] A. Affolder, P.P. Allport, G. Casse, IEEE Trans. Nucl. Sci. 56 (2009) 765

[13] I. Mandic, et al. Nucl. Inst. And Meth. A 612 (2010) 474.

[14] G. Casse, et al. Nucl. Inst. And Meth. A 624 (2010) 401.

[15] G. Casse, et al. Nucl. Inst. And Meth. A 636 (2011) s56.

[16] F. Anghinolfi, et al., IEEE Trans. Nucl. Sci. NS-3 (44) (1997) 298.

[17] V. Eremin, et al., Nucl. Instr. and Meth. A 658 (2011) 145.

[18] G. Kramberger, et al. IEEE Trans. Nucl. Sci. 57 (2010) 2294.

[19] G. Kramberger, et al., presented at 17th RD50 Workshop, CERN, 11/2010

[20] D. Menichelli, M. Bruzzi, Z. Li, and V. Eremin, Nucl. Instr. and Meth. A, 426 (1999) 135.

[21] G. Lindström, M. Moll and E. Fretwurst, Nucl. Inst. And Meth. A 428 (1999) 1. 